ELECTRONIC RESEARCH ANNOUNCEMENTS OF THE AMERICAN MATHEMATICAL SOCIETY

Volume 9, Pages 1-9 (January 10, 2003)

S $1079-6762(03) 00105-7$

\title{
NONPOSITIVE IMMERSIONS, SECTIONAL CURVATURE, AND SUBGROUP PROPERTIES
}

DANIEL T. WISE

(Communicated by Walter Neumann)

\begin{abstract}
This announcement describes research concerning local quasiconvexity, coherence, compact cores, and local indicability for fundamental groups of certain 2-complexes.
\end{abstract}

\section{INTRODUCTION}

Gromov's word-hyperbolic groups have had a profound impact on the theory of infinite groups. For some purposes, however, word-hyperbolicity is far too general a property to work with. Perhaps the single most important motivating example of a word-hyperbolic group is the fundamental group of a genus 2 surface. And yet, general word-hyperbolic groups fail to satisfy one of the most basic properties of a surface group. Namely, every finitely generated subgroup of a surface group is itself a surface group, but not every finitely generated subgroup of a word-hyperbolic group is word-hyperbolic or even finitely presented [26].

A group is coherent if each of its finitely generated subgroups is finitely presented. A significant early result on coherent groups proven independently by Scott and Shalen and applying ideas of Swarup, is that the fundamental group of any 3manifold is coherent [27]. There has recently been a resurgence of interest in the coherence property. Most notably Feighn-Handel proved that free-by-cyclic groups are coherent [10, and McCammond-Wise proved that groups satisfying a certain 'perimeter' condition are coherent 20. The purpose of this announcement is to describe a variety of further results concerning coherence and related properties. In many ways, this is the beginning of a theory offering a more faithful generalization of surface groups than the theory of word-hyperbolic groups.

Section [1] describes my results from [30], where the nonpositive immersion property for 2-complexes is introduced. The fundamental group of a 2-complex with nonpositive immersions is coherent and locally indicable. This is the strongest result on coherent groups to date. It subsumes the Feighn-Handel result on coherence of ascending HNN extensions [10, it subsumes the coherence of finitely generated 3manifold groups with cohomological dimension 2, and it appears likely to subsume the perimeter coherence results given in [20].

Received by the editors October 21, 2002.

2000 Mathematics Subject Classification. Primary 20F05, 20F67, 57M07, 57M20.

Key words and phrases. Coherent groups, nonpositive curvature, one-relator groups.

Research supported by grants from FCAR and NSERC. 
Section 2 describes results from [32, which introduces a new form of sectional curvature for 2-complexes with angles at the corners of their 2-cells. The 2complexes with nonpositive sectional curvature have the nonpositive immersion property, and thus have coherent fundamental groups. The compact 2-complexes with negative sectional curvature have the compact core property for covering spaces with finitely generated fundamental group, and this facilitates further understanding of their subgroups. We emphasize that the sectional curvature notion is a "conformal" theory requiring angles but not requiring a metric. However, when the 2-complex has negative sectional curvature arising from a genuine metric of nonpositive curvature one can prove that the fundamental group is actually locally quasiconvex. These results are parallel to the local quasiconvexity theorem proven for certain small-cancellation groups in 20 .

Finally, Section 3 describes significant progress made in 31 towards Baumslag's long-standing conjecture on the coherence of one-relator groups. I propose a graphtheoretical conjecture that would imply the coherence of all one-relator groups.

\section{NONPOSITIVE IMMERSIONS}

We shall work in the category of combinatorial 2-complexes and combinatorial maps. An immersion is a local injection.

Definition 1.1. A 2-complex $X$ has nonpositive immersions if for every immersion $Y \rightarrow X$ with $Y$ compact, either $\chi(Y) \leq 0$ or $\pi_{1} Y$ is trivial.

Our main theorem is the following surprising coherence result:

Theorem 1.2. If $X$ has nonpositive immersions, then $\pi_{1} X$ is coherent.

A group $G$ is locally indicable if every nontrivial finitely generated subgroup of $G$ has an infinite cyclic quotient. The best known classes of locally indicable groups are torsion-free one-relator groups [4, 5] and fundamental groups of irreducible 3-manifolds with boundary [14]. The following plays a role in the proof of Theorem 1.2.

Theorem 1.3. If $X$ has nonpositive immersions, then $\pi_{1} X$ is locally indicable.

The following is proven through a simple counting argument utilizing the graph of spaces decomposition:

Theorem 1.4. Let $X$ be the standard 2-complex of an ascending HNN extensions of a free groups. Then $X$ has nonpositive immersions.

The following is proven by combining an asphericity result on 3-manifold spines [16, [7] with the fact that the Euler characteristic of a closed 3-manifold is zero:

Theorem 1.5. Every compact irreducible 3-manifold with boundary has a spine with nonpositive immersions.

One of the main tools employed in the proofs of the theorems presented here are towers (see [15]). Towers first appeared explicitly in Papakyriakopolous's proof of Dehn's Lemma [23]. However, the tower method is implicit in Magnus's original solution of the word-problem and Freiheitssatz for one-relator groups [18, 19]. 
Definition 1.6 (Tower). A tower $T \rightarrow X$ is a map which can be expressed as a composition

$$
T=\hat{X}_{n} \rightarrow X_{n} \hookrightarrow \cdots \hookrightarrow \hat{X}_{2} \rightarrow X_{2} \hookrightarrow \hat{X}_{1} \rightarrow X_{1}=X
$$

where each $\hat{X}_{i} \rightarrow X_{i}$ is a covering map, and each $X_{i+1} \hookrightarrow \hat{X}_{i}$ is an inclusion.

We can now briefly sketch the proofs of our Theorems 1.3 and 1.2 .

Sketch of Theorem 1.3. Let $\hat{X}$ be a covering space, and suppose $\mathrm{H}^{1}(\hat{X})=0$. Let $Y \rightarrow \hat{X}$ be a $\pi_{1}$-surjection with $Y$ compact and $\mathrm{H}^{1} Y=0$. Let $Y \rightarrow T \rightarrow \hat{X}$ be a maximal tower lift of $Y \rightarrow \hat{X}$. Then $Y \rightarrow T$ is $\pi_{1}$-surjective, and hence $\mathrm{H}^{1} T=0$, so $\chi(T) \geq 1$, and so $\pi_{1} T$ is trivial. But then $\pi_{1} \hat{X}$ is trivial, since $T \rightarrow \hat{X}$ is $\pi_{1}$-surjective.

Sketch of Theorem 1.2. Consider a finitely generated subgroup $H \subset \pi_{1} X$ presented by

$$
\left\langle a_{1}, \ldots, a_{r} \mid R_{1}, R_{2}, \ldots\right\rangle .
$$

For each $i$ there is a subpresentation $P_{i}=\left\langle a_{1}, \ldots, a_{r} \mid R_{1}, \ldots, R_{i}\right\rangle$, and a maximal tower lift of $P_{i} \rightarrow X$ to $P_{i} \rightarrow T_{i}$. In this way we obtain a sequence $T_{1} \rightarrow T_{2} \rightarrow \cdots$. We choose $m$ such that $\chi\left(T_{m}\right)$ is maximal, and then $T_{m} \rightarrow X$ is $\pi_{1}$-injective, and hence a $\pi_{1}$-isomorphism to $H$. This final step employs Howie's Freiheitssatz for nonsingular systems of equations over locally indicable groups [13].

\section{Sectional Curvature For 2-COMPlexes}

We begin by describing a combinatorial generalization of the Gauss-Bonnet theorem. This was discovered in conjunction with a study of small-cancellation theory 21], but had actually been observed previously in [1. Previous versions of the Gauss-Bonnet theorem were concerned with disc diagrams (i.e. singular surfaces) of one type or another [17, 28, 12, 25]. We shall state the Combinatorial GaussBonnet Theorem after making the following definitions:

The 2-complex $X$ is an angled 2-complex if a real number called an angle is assigned to each corner of each 2-cell of $X$. Equivalently, the angles are assigned to the edges of the links of 0-cells of $X$, and in an obvious sense, each 2-cell and 0-cell has a set of corners with assigned angles. The most natural angled 2-complexes arise by identifying each 2-cell with a Euclidean polygon. The metric, however, plays no role for many of the arguments, and it is often useful to allow zero angles or even negative angles. The curvature $\kappa(v)$ of a 0 -cell $v \in X^{0}$ is defined by

$$
\kappa(v)=2 \pi-\pi \chi(\operatorname{link}(v))-\sum_{c \in \operatorname{Corners}(v)} \angle c .
$$

For a 2-cell $f$ with $|\partial f|$ sides, the curvature $\kappa(f)$ is defined by

$$
\kappa(f)=(|\partial f|-2) \pi-\sum_{c \in \operatorname{Corners}(f)} \angle c .
$$

Theorem 2.1 (Combinatorial Gauss-Bonnet Theorem). Let $X$ be an angled 2complex. Then

$$
2 \pi \chi(X)=\sum_{v \in X^{0}} \kappa(v)+\sum_{f \in 2 \text {-cells }(X)} \kappa(f)
$$


Since formula (11) allows us to measure the curvature of a 0-cell in an arbitrary angled 2-complex, we are able to consider the curvatures of arbitrary sections in $X$, and not just the planar sections. This motivates the following:

Definition 2.2 (Sectional curvature). Let $X$ be an angled 2-complex and let $x \in$ $X^{0}$. A regular section at $x$ is an immersion $(S, s) \rightarrow(X, x)$, where $S$ is a 2complex, and $\operatorname{link}(s)$ is a connected nonempty graph with no vertices of valence $\leq 1$. We say $X$ has nonpositive sectional curvature at $x$ if for every regular section $(S, s) \rightarrow(X, x)$, after pulling back the angles to corners of $S$, the curvature $\kappa(s)$ is nonpositive. We say that $X$ has nonpositive sectional curvature if each 2-cell of $X$ has nonpositive curvature and $X$ has nonpositive sectional curvature at each 0 -cell.

The section $(S, s) \rightarrow(X, x)$ is a planar section if $\operatorname{link}(s)$ is a cycle. We say $X$ has nonpositive planar sectional curvature if each 2-cell has nonpositive curvature and $\kappa(s) \leq 0$ for each $x \in X^{0}$ and each planar section $(S, s) \rightarrow(X, x)$.

We say $X$ has negative [planar] sectional curvature if $\kappa(s)<0$ for each [planar] section $(S, s) \rightarrow(X, x)$. Note that we permit the 2-cells of $X$ to have curvature $\leq 0$ in this case.

2.1. The weight test. The nonpositive planar sectional curvature property is a variation on a usual theme in geometric group theory, and so we shall briefly review and compare similar notions. An angled 2-complex $X$ satisfies the Gersten-Pride nonpositive weight test if for each $x \in X^{0}$, every immersed cycle in $\operatorname{link}(x)$ has angle-length $\geq 2 \pi$, where the angle-length is the sum of the angles of the edges traversed by the cycle [12 25. Similarly, the negative weight test requires that the angle-length be $>2 \pi$. Among the many interesting weight test consequences is that $\pi_{1} X$ is word-hyperbolic provided that $X$ is compact and satisfies the negative weight test. Note that if $X$ has no negative angles, then $X$ satisfies the nonpositive [negative] weight test if and only if it has nonpositive [negative] planar sectional curvature.

The foremost examples of 2-complexes satisfying the weight test are piecewise Euclidean 2-complexes with a metric of nonpositive curvature. These are formed by gluing together a set of Euclidean polygons along their sides, so that the gluing maps are isometries, and such that the nonpositive weight test is satisfied. A finiteness condition is also necessary, and we refer the reader to 24] for a lucid and concise introduction to metric spaces of nonpositive curvature, and to [3] for a more recent extensive treatment.

Because their angles are nonnegative, piecewise Euclidean 2-complexes have metrics of nonpositive curvature exactly when they have nonpositive planar sectional curvature. However, nonpositive sectional curvature is considerably more sensitive. For instance, let $B_{n}$ denote a bouquet of $n$ circles, let $X_{n}=B_{n} \times B_{n}$ be the piecewise Euclidean 2-complex whose 2-cells are unit squares, and let $x$ denote the unique 0-cell in $X_{n}$. Since $\operatorname{link}(x)$ is a complete bipartite graph and each angle is $\frac{\pi}{2}$, it is easy to see that $X_{n}$ satisfies the nonpositive weight test. However, $\kappa(x)>0$ if $n>2$, so while $X_{n}$ has nonpositive planar sectional curvature, it does not have nonpositive sectional curvature.

2.2. Consequences of nonpositive and negative sectional curvature. We first observe that nonpositive sectional curvature implies the nonpositive immersion property because of the following: 
Theorem 2.3. Let $X$ have nonpositive sectional curvature. Let $Y \rightarrow X$ be an immersion where $Y$ is compact and connected. Then either $\chi(Y) \leq 0$ or $Y$ deformation retracts to a single 0 -cell.

Sketch. We first repeatedly collapse along free faces to deformation retract to a subcomplex $Z$ of $Y$ such that $Z$ has no free faces and $\chi(Z)=\chi(Y)$. Now suppose that $Z$ does not consist of a single 0-cell, and pull back the angles of $X$ to the corners of $Z$. For each $z \in Z^{0}$, either $\operatorname{link}(z)$ consists of two or more vertices, and so $\kappa(z) \leq 0$ or $\operatorname{link}(z)$ contains an edge, and so $\kappa(z) \leq 0$ because of the nonpositive sectional curvature hypothesis. Therefore $\chi(Z) \leq 0$ by Theorem 2.1 .

Our two main results about 2-complexes with negative sectional curvature are Theorem 2.7 below and the following analogue of the compact core theorem for 3-manifolds:

Theorem 2.4 (Compact Core). Let $X$ have negative sectional curvature. Let $\hat{X} \rightarrow X$ be a covering space such that $\pi_{1} \hat{X}$ is finitely generated. Then there is a compact subcomplex $C \subset \hat{X}$ such that $C \rightarrow \hat{X}$ induces a $\pi_{1}$-isomorphism.

One immediate algebraic consequence of Theorem 2.4 is that if $X$ has negative sectional curvature, then $\pi_{1} X$ is coherent. The proof of Theorem 2.4 is substantially less complex than the proof of Theorem 1.2 It would be desirable to have a similar direct proof of coherence from the hypothesis of nonpositive sectional curvature. However, there are 2-complexes described in [34 that do not have the compact core property, and it is shown in [32] that angles can be assigned so that these 2complexes have nonpositive sectional curvature. Nevertheless, some of those angles are negative, so there is hope of generalizing Theorem 2.4 to the case where $X$ has nonnegative or positive angles.

We note that not every word-hyperbolic group is coherent. The first counterexamples were small-cancellation groups found by Rips in [26. Examples of compact 2 -complexes with metrics of negative curvature but with incoherent fundamental group were given in 33 . In many respects, the fundamental groups of 2-complexes with negative sectional curvature behave very much like those of genus 2 surfaces. This is further supported by the following consequences of Theorem 2.4.

Corollary 2.5 (Deficiency). Let $X$ have nonpositive sectional curvature, and suppose each 2-cell has curvature $\leq-2 \pi \epsilon<0$. Any subgroup of $\pi_{1} X$ generated by $r$ elements has a presentation with at most $\frac{r-1}{\epsilon}$ relators.

In particular, Corollary [2.5 implies that all sufficiently low rank subgroups are free.

Corollary 2.6 (Finitely many conjugacy classes). Let $X$ be a compact angled 2complex with negative sectional curvature. For each $r$, there are finitely many conjugacy classes of noncyclic freely indecomposable subgroups of $\pi_{1} X$ generated by $r$ elements.

We now describe a more powerful result about 2-complexes with negative sectional curvature. It has a metric conclusion and requires a stronger metric hypothesis. Our actual result is more general but involves a technical condition involving the existence of certain "straight lines" that we shall not state here.

Theorem 2.7 (Local Quasiconvexity). Let $X$ be a compact 2-complex with a piecewise Euclidean metric of nonpositive curvature. 
If $X$ has negative sectional curvature, then $\pi_{1} X$ is locally quasiconvex.

2.3. Examples with nonpositive and negative sectional curvature. The results described above suggest that the class of spaces with negative sectional curvature is very limited. However, this class coincides quite accurately with many of the spaces that have traditionally occupied the central stage. We shall now describe a variety of such examples.

By taking the dual 2-complex to the ideal triangulation obtained in [9] we have the following:

Theorem 2.8. Let $M$ be a finite volume cusped hyperbolic 3-manifold. Then $M$ has a spine with nonpositive sectional curvature.

One of our most intriguing classes of examples relates the usual nonpositive planar sectional curvature with the most basic 3-manifold property.

Theorem 2.9. Let $X$ have nonpositive [negative] planar sectional curvature. Suppose that $\operatorname{link}(x)$ embeds in $S^{2}$ for all $x \in X$. Then $X$ has nonpositive [negative] sectional curvature.

In fact, a more general version of the above theorem holds with the assumption that lowlink $(x)$ be spherical, where $\operatorname{low} \operatorname{link}(x)$ is the subgraph of $\operatorname{link}(x)$ containing all edges corresponding to corners with angle $<\pi$. This leads to the following two well-known classes that fit into this framework.

The first yields a class of ascending HNN extensions of free groups which are fundamental groups of 2-complexes with nonpositive sectional curvature. Note that despite Theorem 1.4 it seems unlikely that every ascending HNN extension of a free group arises as $\pi_{1} X$, where $X$ has nonpositive sectional curvature.

Theorem 2.10. Let $B$ be a bouquet of circles, and $\phi: B \rightarrow B$ be an immersion. Let $X$ be the 2-complex for the mapping torus of $\phi$. Then $X$ has nonpositive sectional curvature.

Adian's "cycle-free" presentations, which were studied in 29] and [12, are presentations of the form

$$
\left\langle a_{1}, a_{2}, \cdots \mid U_{1}=V_{1}, U_{2}=V_{2}, \ldots\right\rangle,
$$

where $U_{i}$ and $V_{i}$ are positive words for each $i$, and the ascending and descending links are forests. These are the largest subgraphs of the link containing only the $a_{i}^{+1}$ and only the $a_{i}^{-1}$ vertices, respectively.

Theorem 2.11. Let $X$ be the standard 2-complex of an Adian presentation. Then $X$ has nonpositive sectional curvature.

In particular, it follows from Theorem 1.3 that Adian groups are locally indicable and hence left-orderable by [6], which answers a question raised in [29]. Following a suggestion of Stallings, it was observed in [12] that Adian presentations satisfy Sieradski's asphericity test [28], which is equivalent to the Gersten-Pride weight test where only $\pi$ and 0 angles are allowed. In fact, we prove that every 2-complex satisfying Sieradski's weight test has nonpositive sectional curvature!

Our final family of examples of 2-complexes gives some further sense of the scope of nonpositive sectional curvature. We say $X$ is a $(p, q, r)$ complex provided each 2-cell has $\geq p$ sides, each cycle in $\operatorname{link}(x)$ has length $\geq q$ for all $x \in X^{0}$, and each 1 -cell appears $\leq r$ times on the boundary of 2-cells. 


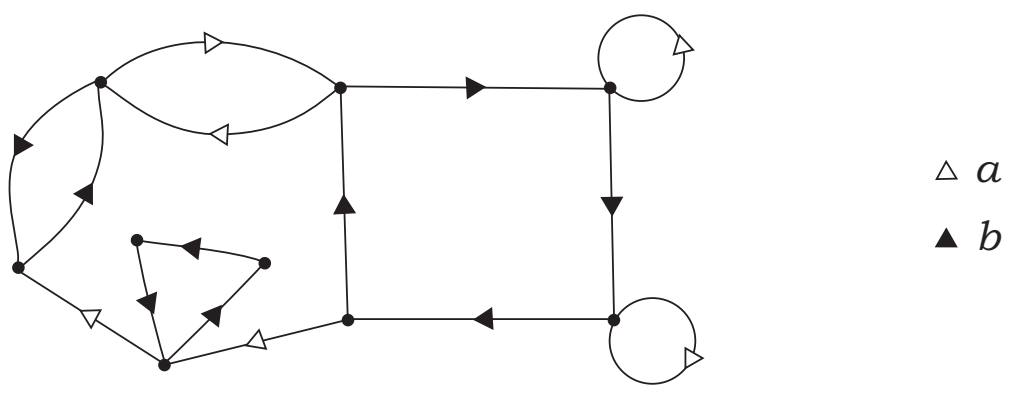

FIGURE 1.

Theorem 2.12. Let $X$ be $a(p, q, r)$ complex with $p \geq \frac{q}{q-2} r$. Assign an angle of $\frac{(p-2)}{p} \pi$ to each corner. Then $X$ has nonpositive curvature, and $X$ has negative curvature if the inequality is sharp.

\section{ONE-RELATOR GROUPS}

Gilbert Baumslag raised the following in [2]:

Conjecture 3.1. Every group defined by a presentation with a single defining relation is coherent.

Let $X$ be the standard 2-complex of a one-relator presentation $\left\langle a_{1}, a_{2}, \cdots \mid W\right\rangle$, and suppose that $W$ is freely and cyclically reduced. If $W$ is a proper power, then let $\hat{X}$ be a based cover corresponding to a torsion-free finite index subgroup [11, and let $X^{\prime}$ be an aspherical $\pi_{1}$-isomorphic subcomplex of $\hat{X}$ obtained by removing duplicate 2-cells. By applying Theorem 1.2 we hope to prove Conjecture 3.1 by showing that:

Conjecture 3.2. If $W$ is not a proper power, then $X$ has nonpositive immersions.

If $W$ is a proper power, then $X^{\prime}$ has nonpositive immersions.

This conjecture has a lovely formulation in terms of certain paths in a labeled oriented graph $\Gamma$ which is a connected graph whose edges are directed and labeled by letters in some alphabet set $\left\{a_{1}, a_{2}, \ldots\right\}$ and which satisfies the following condition: No vertex has two incoming edges with the same label, and no vertex has two outgoing edges with the same label. A combinatorial path $P \rightarrow \Gamma$ starting at some vertex $v$ determines a word in $\left\{a_{1}^{ \pm 1}, a_{2}^{ \pm 1}, \ldots\right\}$. Conversely, a word $W$ in $\left\{a_{1}^{ \pm 1}, a_{2}^{ \pm 1}, \ldots\right\}$ determines at most one path $P$ starting at a vertex $v$. A $W$-cycle is a closed path corresponding to the word $W$. If $W=V^{n}$, then we shall not distinguish between two $W$-cycles if their starting vertices are connected by a path corresponding to a word $V^{i}$ for some $i$. For instance, the graph in Figure 1 has exactly three distinct $a^{2}$-cycles, no $b$-cycles, two $b^{4}$-cycles, three $b^{12}$-cycles, three $a a b a^{-1} b^{-1}$-cycles, and no $a b$-cycles.

Conjecture 3.3. Let $\Gamma$ be a labeled oriented graph. Then the number of $W$-cycles in $\Gamma$ is at most $\beta_{1}(\Gamma)$. In particular, when $\Gamma$ is connected, the number of $W$-cycles is bounded by $1+e-v$, where $e$ and $v$ are the numbers of edges and vertices in $\Gamma$.

Here are two additional algebraic formulations in terms of subgroups of free groups. 
Conjecture 3.4. Let $H$ be a subgroup of $F$, and let $Z$ be a cyclic subgroup of $F$. Then the number of distinct conjugates of $Z$ that intersect $H$ nontrivially is bounded by $\operatorname{rank}(H)$.

Readers familiar with Walter Neumann's generalized Hanna Neumann conjecture (see [22, 8]) will note that Conjecture 3.4 bears intriguing similarity with a 'rank 1 variant'. Unfortunately, the Hanna Neumann conjecture carries no information when one of the subgroups has rank 1, and the standard approach towards the Hanna Neumann conjecture does not immediately lead to a proof of Conjecture 3.4 Nevertheless, hopefully some fruitful connection will eventually be realized.

A subgroup $H \subset F$ is isolated if $g^{n} \in H-\left\{1_{F}\right\}$ implies that $g \in H$. When $W$ is not a proper power, Conjecture 3.3 can also be interpreted as follows:

Conjecture 3.5. Let $H$ be an isolated rank $r$ subgroup of a free group $F$. Let $H_{1}, \ldots, H_{r+1}$ be distinct conjugates of $H$ in $F$. Then $\bigcap_{i=1}^{r+1} H_{i}=\left\{1_{F}\right\}$.

Thus far, we have been able to prove Conjecture 3.2 in several cases. For example:

Theorem 3.6. Suppose the one-relator group $\left\langle a_{1}, a_{2}, \cdots \mid W\right\rangle$ is residually torsionfree solvable. Then $X$ has nonpositive immersions.

A positive one-relator group is a one-relator group such that for some generator $a_{i}$, the relator $W$ contains $a_{i}$ but does not contain $a_{i}^{-1}$. Of course, the motivating case is when $W$ is a positive word in the generators.

Theorem 3.7. Let $X$ be the standard 2-complex of a positive one-relator group. Then $X$ [respectively $\left.X^{\prime}\right]$ has nonpositive immersions.

As for many results in the theory of one-relator groups, the proofs employ staggered 2-complexes [15], and the analogous theorems actually hold for staggered 2 -complexes.

\section{REFERENCES}

[1] W. Ballmann and S. Buyalo. Nonpositively curved metrics on 2-polyhedra. Math. Z., 222(1):97-134, 1996. MR 97c:53063

[2] Gilbert Baumslag. Some problems on one-relator groups. In Proceedings of the Second International Conference on the Theory of Groups (Australian Nat. Univ., Canberra, 1973), volume 372 of Lecture Notes in Math., pages 75-81. Springer, Berlin, 1974. MR 51:717

[3] Martin R. Bridson and André Haefliger. Metric spaces of non-positive curvature. SpringerVerlag, Berlin, 1999. MR 2000k:53038

[4] S. D. Brodskiı̌. Equations over groups and groups with one defining relation. Uspekhi Mat. Nauk, 35(4(214)):183, 1980. MR 82a:20041

[5] S. D. Brodskir. Equations over groups, and groups with one defining relation. Sibirsk. Mat. Zh., 25(2):84-103, 1984. MR 86e:20026

[6] R. G. Burns and V. W. D. Hale. A note on group rings of certain torsion-free groups. Canad. Math. Bull., 15:441-445, 1972. MR 46:9149

[7] J. M. Corson and B. Trace. Diagrammatically reducible complexes and Haken manifolds. J. Austral. Math. Soc. Ser. A, 69(1):116-126, 2000. MR 2001f:57005

[8] Warren Dicks. Equivalence of the strengthened Hanna Neumann conjecture and the amalgamated graph conjecture. Invent. Math., 117(3):373-389, 1994. MR 95c:20034

[9] D. B. A. Epstein and R. C. Penner. Euclidean decompositions of noncompact hyperbolic manifolds. J. Differential Geom., 27(1):67-80, 1988. MR 89a:57020

[10] Mark Feighn and Michael Handel. Mapping tori of free group automorphisms are coherent. Ann. of Math. (2), 149(3):1061-1077, 1999. MR 2000i:20050

[11] J. Fischer, A. Karrass, and D. Solitar. On one-relator groups having elements of finite order. Proc. Amer. Math. Soc., 33:297-301, 1972. MR 47:342 
[12] S. M. Gersten. Reducible diagrams and equations over groups. In Essays in group theory, pages 15-73. Springer, New York-Berlin, 1987. MR 89d:20030

[13] James Howie. On pairs of 2-complexes and systems of equations over groups. J. Reine Angew. Math., 324:165-174, 1981. MR 82g:20060

[14] James Howie. On locally indicable groups. Math. Z., 180(4):445-461, 1982. MR 84b:20036

[15] James Howie. How to generalize one-relator group theory. In S. M. Gersten and John R. Stallings, editors, Combinatorial group theory and topology, pages 53-78, Princeton, N.J., 1987. Princeton Univ. Press. MR 88j:20027

[16] Johannes Huebschmann. Aspherical 2-complexes and an unsettled problem of J. H. C. Whitehead. Math. Ann., 258(1):17-37, 1981/82. MR 83e:57004

[17] Roger C. Lyndon and Paul E. Schupp. Combinatorial group theory. Springer-Verlag, Berlin, 1977. Ergebnisse der Mathematik und ihrer Grenzgebiete, Band 89. MR 58:28182

[18] Wilhelm Magnus. Über diskontinuierliche Gruppen mit einer definierenden Relation (Der Freiheitssatz). J. Reine Angew. Math., 163:141-165, 1930.

[19] Wilhelm Magnus. Das Identitätsproblem für Gruppen mit einer definierenden Relation. Math. Ann., 106:295-307, 1932.

[20] Jonathan P. McCammond and Daniel T. Wise. Coherence, local quasiconvexity and the perimeter of 2-complexes. Preprint, 1999.

[21] Jonathan P. McCammond and Daniel T. Wise. Fans and ladders in small cancellation theory. Proc. London Math. Soc. (3), 84(3):599-644, 2002.

[22] Walter D. Neumann. On intersections of finitely generated subgroups of free groups. In Groups-Canberra 1989, pages 161-170. Springer, Berlin, 1990. MR 92b:20026

[23] C. D. Papakyriakopoulos. On Dehn's lemma and the asphericity of knots. Ann. of Math. (2), 66:1-26, 1957. MR 19:761a

[24] Frédéric Paulin. Constructions of hyperbolic groups via hyperbolizations of polyhedra. In Group theory from a geometrical viewpoint (Trieste, 1990), pages 313-372. World Sci. Publishing, River Edge, NJ, 1991. MR 93d:57005

[25] Stephen J. Pride. Star-complexes, and the dependence problems for hyperbolic complexes. Glasgow Math. J., 30(2):155-170, 1988. MR 89k:20049

[26] E. Rips. Subgroups of small cancellation groups. Bull. London Math. Soc., 14(1):45-47, 1982. MR 83c:20049

[27] G. P. Scott. Finitely generated 3-manifold groups are finitely presented. J. London Math. Soc. (2), 6:437-440, 1973. MR 52:1660

[28] Allan J. Sieradski. A coloring test for asphericity. Quart. J. Math. Oxford Ser. (2), 34(133):97-106, 1983. MR 84g:57004

[29] John R. Stallings. Adian groups and pregroups. In Essays in group theory, pages 321-342. Springer, New York, 1987. MR 88m:20075

[30] Daniel T. Wise. Coherence, local indicability, and non-positive immersions. Preprint 2002.

[31] Daniel T. Wise. Positive one-relator groups are coherent. Preprint 2002.

[32] Daniel T. Wise. Sectional curvature and local quasiconvexity. Preprint 2002.

[33] Daniel T. Wise. Incoherent negatively curved groups. Proc. Amer. Math. Soc., 126(4):957964, 1998. MR 98f:20016

[34] Daniel T. Wise. A covering space with no compact core. Geom. Ded., 92(1):59-62, 2002.

Department of Mathematics, McGill University, Montreal, Quebec, CA H3A 2K6, CANADA

E-mail address: wise@math.mcgill.ca 